Mens

revue d'histoire intellectuelle de l'Amérique française

Louise Bienvenue. Quand la jeunesse entre en scène. L'Action catholique avant la Révolution tranquille. Montréal, Boréal, 2003. 291 p.

\title{
Dominique Marshall
}

Volume 4, numéro 2, printemps 2004

URI : https://id.erudit.org/iderudit/1024603ar

DOI : https://doi.org/10.7202/1024603ar

Aller au sommaire du numéro

Éditeur(s)

Centre de recherche en civilisation canadienne-française

ISSN

1492-8647 (imprimé)

1927-9299 (numérique)

Découvrir la revue

Citer ce compte rendu

Marshall, D. (2004). Compte rendu de [Louise Bienvenue. Quand la jeunesse entre en scène. L'Action catholique avant la Révolution tranquille. Montréal, Boréal, 2003. 291 p.] Mens, 4(2), 344-348. https://doi.org/10.7202/1024603ar d'utilisation que vous pouvez consulter en ligne.

https://apropos.erudit.org/fr/usagers/politique-dutilisation/ 


\section{Louise Bienvenue. Quand la jeunesse entre en scène. L'Action catholique avant la Révolution tranquille. Montréal, Boréal, 2003. 291 p.}

Entre 1930 et 1960, un large mouvement social a traversé le Québec francophone au nom de la jeunesse. Importé de Belgique, un modèle catholique d'action sociale laïque a pu se répandre dans la province de la Grande dépression grâce à un contexte particulièrement favorable et à l'encouragement de Rome et des évêques locaux. Sur le plan des idées, l'injonction papale d'éloignement de la politique, une réaction à l'intolérance du gouvernement fasciste italien, rencontra une audience canadienne-française fatiguée du nationalisme dominant les associations offertes aux jeunes depuis le début du siècle. Pratiquement, les services d'emploi, d'orientation professionnelle et de formation continue des nouvelles organisations offrirent un espoir aux victimes de la Crise et leur concentration dans les villes répondit aux mouvements de la jeune population. La progression générale de la scolarité, la montée des effectifs des classes moyennes, contribuèrent à leur tour à donner davantage de temps libre aux individus en transition entre l'enfance et l'âge adulte.

En promouvant une relative autonomie vis-à-vis des structures paroissiales, en assurant une place accrue dans les instances de direction et en mettant l'accent sur l'apostolat, la récréation, les débats et la spontanéité, autant d'éléments qui avaient assuré la popularité des initiatives belges, l'effort de reconquête de Rome permit à des énergies neuves de prendre forme. Une méthode établissant l'enquête comme base de l'action, selon la formule "voir, juger, agir ", autorisa la formation d'un savoir précis sur la jeunesse "normale", souvent révélateur, produit dans le langage scientifique courant des sphères publiques contemporaines. 
Enfin, l'arrimage à un mouvement international dynamique a donné l'occasion à plusieurs d'apprendre à connaître d'autres cultures, de s'ouvrir à des courants de pensée critiques tels la philosophie personnaliste française des années 1930 et, en quelques occasions, de visiter des pairs d'autres continents.

L'originalité de l'étude de Louise Bienvenue vient de la lecture concurrente des publications de l'ensemble des jeunes organisations d'action catholique entre 1930 et 1950 . La possibilité de recourir aux monographies récentes portant sur des associations spécifiques ajoute à la profondeur du projet, parmi lesquelles figure le travail sur la Jeunesse agricole catholique que l'auteur lui-même a mené au préalable, sous la supervision d'historiens européens. De nombreuses photographies provenant des archives du mouvement illustrent l'ouvrage ; on regrette seulement que l'auteur ne se soit pas attardé davantage aux éléments de ces représentation visuelles de la jeunesse et n'ait pas tenté d'en interroger le sens.

L'analyse de la variété des regroupements spécialisés permet aussi de mesurer l'influence des distinctions de sexe et de classe sur l'engagement des jeunes et sur leur identité. Les techniques de l'apostolat laic, par exemple, initièrent plusieurs jeunes femmes à de nouvelles fonctions. Paradoxalement, la spécialisation entre agriculteurs, ouvriers, étudiants et « indépendants » porta plusieurs individus à réfléchir sur la place et les obligations respectives des membres de leur groupe. Dans certaines circonstances, une telle structure, inspirée du corporatisme catholique, a pu favoriser les échanges entre classes, de même qu'un certain respect mutuel. En même temps, l'ensemble des organisations s'entendit pour revendiquer la prolongation des études, l'accroissement des prérogatives publiques des jeunes et, après la guerre, le pacifisme. 
L'auteur identifie les préoccupations des membres et leurs actions en montrant par exemple que les "usages sociaux de l'âge » (pp. 72, 264) furent eux-mêmes l'objet de luttes internes. Elle fait aussi apparaitre les transformations des engagements, qu'elle regroupe en deux périodes séparées par la Seconde Guerre mondiale. Ce faisant, elle se démarque des lectures de la culture politique qui tendent uniquement vers l'explication de la libéralisation des années 1960. Les années d'entre-deux-guerres représentent le point culminant de la popularité d'un mouvement qui tenta continuellement de rejoindre les masses.

Quand la jeunesse entre en scène s'inscrit dans un courant historiographique soucieux de considérer les "générations" comme des "constructions sociales", avec le même sens critique qui anime les études sur les distinctions entre les sexes, les classes sociales et les identités ethniques et nationales. Bienvenue peut ainsi montrer qu'au fil des transformations de la conjoncture, la recherche de légitimité a renouvelé le sens même de la notion de jeunesse. Avant 1940, il s'agit d'un groupe pur, indépendant et vrai, d'une " génération sacrifiée " par la Crise, visant à l'héroïsme, mais toujours respectueuse la « hiérarchie d'âge » (p. 81). Dans les années 1940, devant la perte d'estime du corporatisme, devant la multiplication des institutions publiques du nouvel État-providence interpellant les jeunes, la jeunesse se présenta davantage comme un regroupement partisan et expert, plus individualiste, expressif et personnel. Elle devait agir davantage comme un groupe de pression, selon les principes d'un « humanisme libéral » (p. 166). Dans les années 1950, elle fut aussi affaiblie par le renforcement général de l'autorité des évêques.

Les nuances mises à jour par cette réflexion sur les constructions sociales de générations invitent à l'étude d'une nouvelle série de questions. Comment relier ces jeunes à leurs familles? Comment les transformations subséquentes des di- 
rectives pontificales ont-elles influencé les associations que Rome avait contribué à fonder ? En quoi leurs actions ontelles favorisé les transformations subséquentes du mouvement ? Quel rôle le Québec francophone a-t-il joué dans l'histoire de l'ensemble du mouvement catholique d'action sociale ? Dans quelle mesure les inquiétudes du mouvement local de l'après-guerre sur la nature de l'action catholique se retrouvèrent-elles en Europe? Furent-elles résolues de manières comparables outre-mer?

La plus importante de ces interrogations est probablement celle des façons dont les jeunes de l'entre-deux-guerres ont pu réconcilier les notions d'apolitisme et de démocratie. En adoptant avec enthousiasme l'idée d'un cantonnement aux sphères culturelle et sociale, ils prétendaient qu'ils occupaient une place privilégiée pour la critique des institutions publiques contemporaines auxquelles ils n'auraient accès que plus tard. Ils dénoncèrent ainsi la futilité du mythe de la «mort héroïque " relié à la conscription (p. 88), l' « inertie » du monde des adultes (p. 104) ou encore la corruption du système électoral. Dès lors, comment allait s'accomplir leur pleine prise en charge des responsabilités publiques, une fois entrés dans l'âge adulte ? Allaient-ils continuer à rejeter les maux de leur société sur les générations précédentes ? Si oui, dans quelle mesure croyaient-ils que, d'une génération à l'autre, les sociétés peuvent se départir des responsabilités que leurs prédécesseurs ont reçues de l'histoire et des traditions ?

Leur pensée sur le phénomène de la transmission entre générations des traditions sociales dont leurs parents avaient eux-mêmes hérité, au-delà de vagues déclarations reconnaissantes des obligations des citoyens, était lacunaire. Cet inachèvement n'est peut-être pas étranger au fait que de nombreuses têtes dirigeantes des mouvements d'action catholiques sont restées longtemps "jeunes ", une fois mariées ou bien une fois entrées dans le marché du travail. Il semble aussi 
relié à la tendance au retrait et à la dérision que certains auteurs des journaux de jeunes purent afficher devant les difficultés d'ouvrir leurs actions et leurs réflexions à d'autres groupes de jeunes. Les dénonciations du communisme, par exemple, s'accompagnèrent rarement d'une justification. Pareillement, Maurice Sauvé continua de représenter le Canada à des rencontres internationales du tournant des années 1950 alors qu'il était incapable de recueillir le support des groupes francophones et anglophones officiellement nécessaires.

En épilogue, Louise Bienvenue montre que ses conclusions appellent une relecture de la « seconde poussée associative » des années 1960. Son étude, écrit-elle avec raison, montre qu'il s'agit de «foyers d'affirmation » autres, d'actions d'un genre souvent nouveau, où la "figure changeante de l'étudiant universitaire » a pu peser davantage.

Dominique Marshall

Chercheur invité

Département d'bistoire

Oxford-Brookes University

Jean-François de Raymond. Descartes et le Nouveau Monde. Le cheminement du cartésianisme au Canada XVII $-X X^{e}$ siècle. Paris/ Québec, Librairie philosophique J. Vrin/Presses de l'Université Laval, 2003. 333 pages (collection Zêtêsis, série " Textes et essais ").

Cet ouvrage très documenté retrace minutieusement l'évolution de l'enseignement du cartésianisme au Canada, du XVII ${ }^{e}$ siècle au $\mathrm{XX}^{\mathrm{e}}$. Il n'y est guère question de René 